\title{
Spotlight on tumor mutational burden in patients with non-small cell lung carcinoma
}

In the last recent years, immune checkpoint inhibitors directed against programmed cell death-1 (PD-1) and its ligand programmed death-ligand 1 (PD-L1), have strongly changed the treatment paradigm of patients with non-small cell lung cancer (NSCLC). After initial clinical trials demonstrated improved outcome with these agents in patients with advanced or metastatic NSCLC, immunotherapy now has demonstrated survival advantage in some patients with early-stage NSCLC $(1,2)$. Recent data suggest that combining these agents with chemotherapy or other immune checkpoint inhibitors may improve survival in patients with metastatic NSCLC compared to chemotherapy alone (3). However, the anti-PD-1/PD-L1 inhibitors have demonstrated highly durable responses in only a minority of patients. The modest impact of PD-L1 tumor expression to identify predictors of response to these agents has prompted the search for additional predictive biomarkers to better select patients who will benefit from immunotherapy (4).

In the rapidly evolving landscape of emerging biomarkers for immunotherapy, tumor mutational burden (TMB) is very promising as a predictive biomarker and potentially could lead the way for precision immuno-oncology. In this context, this focused issue of Translational Lung Cancer Research (TLCR) aims to capture the progress, challenges, limitations and perspectives to understand the measurement methods and clinical significance of TMB in patients with lung carcinoma.

In principle, tumor cells are genetically unstable and harbor high levels of somatic mutations that can result in the expression of neoantigens (5). Nonetheless, the prevalence of somatic mutations is considerably variable between different types of solid tumor, with NSCLC having among the highest mutation frequency (6). As such, tumors with high non-synonymous TMB express large numbers of abnormal proteins, which are recognized by the immune system as neoantigens (7). These tumors may be eligible to successful immune-mediated destruction if their T-cell-dependent immune responses against tumor cells could be overreached (8). Whole exome sequencing (WES) is a comprehensive method for assessing TMB and can identify predicted neoantigens. Despite the proven utility of WES in measuring TMB and predicting response to PD-1/ PD-L1 blockade, it has some limitations such as the cost, the large input DNA amount, as well as the workflow and analysis complexity. In parallel, a number of methods have been developed to compute TMB using targeted next-generation sequencing (NGS) panels. The reliability of targeted NGS panels to predict the response to PD-1/PD-L1 blockade encounters several challenges before definite transfer to clinical routine practice such as the size of the panels, the cutoff for high TMB definition, the tissue requirements that usually surpasses the quantity of material obtained from small tissue biopsies, various testing platforms, the need for robust analytical validation, the relatively long turnaround time, wide access in all hospitals, and finally the cost. Assessment of TMB in cell-free DNA in peripheral blood was recently shown to be predictive of survival advantage from immune checkpoint blockade in NSCLC patients (9). While these latter findings need confirmation, this approach could improve the feasibility of assessment of TMB.

This Focused Issue is composed of original research studies and reviews that highlight advances in the field of TMB and lung cancer immunotherapy but also orientate to the new challenges and unanswered questions in this field. We hope that this collection will be of great interest to physicians, pathologists, biologists and researchers in a diverse range of fields.

\section{Acknowledgements}

None.

\section{References}

1. Antonia SJ, Villegas A, Daniel D, et al. Durvalumab after Chemoradiotherapy in Stage III Non-Small-Cell Lung Cancer. N Engl J Med 2017;377:1919-29.

2. Forde PM, Chaft JE, Smith KN, et al. Neoadjuvant PD-1 Blockade in Resectable Lung Cancer. N Engl J Med 2018;378:1976-86.

3. Hellmann MD, Rizvi NA, Goldman JW, et al. Nivolumab plus ipilimumab as first-line treatment for advanced non-small-cell 
lung cancer (CheckMate 012): results of an open-label, phase 1, multicohort study. Lancet Oncol 2017;18:31-41.

4. Steuer CE, Ramalingam SS. Tumor Mutation Burden: Leading Immunotherapy to the Era of Precision Medicine? J Clin Oncol 2018;36:631-2.

5. Gubin MM, Artyomov MN, Mardis ER, et al. Tumor neoantigens: building a framework for personalized cancer immunotherapy. J Clin Invest 2015;125:3413-21.

6. Lawrence MS, Stojanov P, Polak P, et al. Mutational heterogeneity in cancer and the search for new cancer-associated genes. Nature 2013;499:214-8.

7. Braun DA, Burke KP, Van Allen EM. Genomic Approaches to Understanding Response and Resistance to Immunotherapy. Clin Cancer Res 2016;22:5642-50.

8. Rizvi NA, Hellmann MD, Snyder A, et al. Cancer immunology. Mutational landscape determines sensitivity to PD-1 blockade in non-small cell lung cancer. Science 2015;348:124-8.

9. Khagi Y, Goodman AM, Daniels GA, et al. Hypermutated Circulating Tumor DNA: Correlation with Response to Checkpoint Inhibitor-Based Immunotherapy. Clin Cancer Res 2017;23:5729-36.

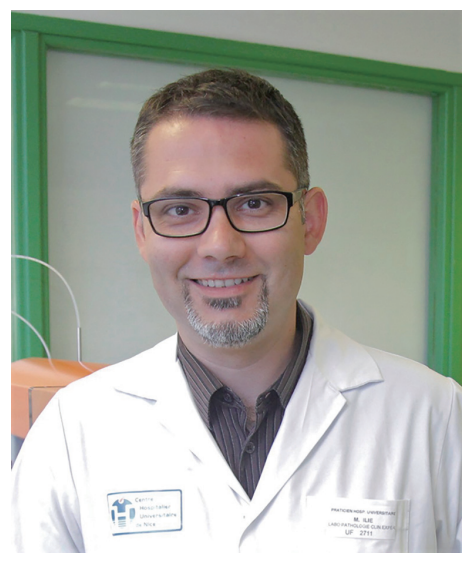

Marius Ilie

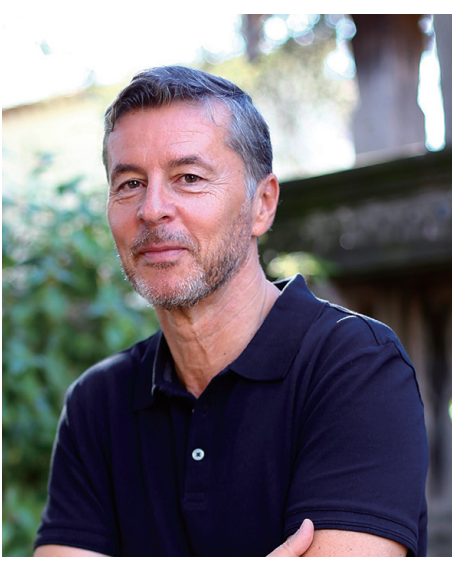

Paul Hofman

\begin{abstract}
Marius Ilie $\mathrm{i}^{1,2,3}$
(Email: ilie.m@chu-nice.fr)

Paul Hofman ${ }^{1,2,3}$

(Email: hofman.p@chu-nice.fr)

${ }^{1}$ Laboratory of Clinical and Experimental Pathology, ${ }^{2}$ Hospital-Integrated Biobank (BB-0033-00025), Pasteur Hospital, University Hospital Federation OncoAge, Université Côte d'Azur, Nice, France; ${ }^{3}$ Institute for Research on Cancer and Aging in Nice (Inserm U1081 and CNRS 7284), Université Côte d'Azur, Nice, France doi: $10.21037 /$ tlcr.2018.08.06 Conflicts of Interest: The authors have no conflicts of interest to declare. View this article at: http://dx.doi.org/10.21037/tlcr.2018.08.06
\end{abstract}

Cite this article as: Ilie M, Hofman P. Spotlight on tumor mutational burden in patients with non-small cell lung carcinoma. Transl Lung Cancer Res 2018;7(6):614-615. doi: 10.21037/ tlcr.2018.08.06 